\title{
UVEITIS PARASITARIA: CASO CLÍNICO EN EL HOSPITAL TEODORO MALDONADO CARBO, 2015
}

\author{
PARASTITC UVEITIS: A CLINICAL CASE IN THE HOSPITAL TEODORO MALDONADO CARBO, 2015
}

UVEÍTE PARASITÁRIA: CASO CLÍNICO NO HOSPITAL TEODORO MALDONADO CARBO, 2015

\section{ROBERTY YUNIOR MEJÍA BERMEO, MARÍA CAMILA CHIQUITO FREILE, ANDREA ALEXANDRA PALMA RUGEL}

Hospital Teodoro Maldonado Carbo, Guayaquil, Ecuador

Resumen

La uveítis es la patología del tracto uveal que describe la inflamación de la úvea en sus tres segmentos: anterior, medio, posterior o panuveitis. Dentro de las uveítis infecciosas se encuentran las causadas por infestaciones parasitarias, las cuales son endémicas en nuestro país. El diagnóstico se lo hace mediante una buena anamnesis, examen físico completo y valoración oftalmológica basada en la agudeza visual, biomicroscopía con lámpara de hendidura y fondo de ojo para poder determinar la localización del parásito. No existe un tratamiento específico para dicha patología, siendo la única forma efectiva, aunque difícil y no siempre posible, la extracción quirúrgica del parásito. A continuación se presenta un caso clínico de una paciente de sexo femenino quien consulta por edema de hemicara izquierda acompañado de edema periorbitario izquierdo y baja visión del ojo del mismo lado, a la cual se le realizó un examen oftalmológico completo y se encontró la presencia de un parásito tubular adherido a la capsula anterior del cristalino del ojo afectado. Se instauró tratamiento médico y se realizó la extracción quirúrgica del parásito.

PALABRAS CLAVE: uveítis, visión reducida, parásitos. veitis is the pathology of the uveal tract that describes the inflammation of the uvea in its three segments: anterior, middle, posterior or panuveitis. Within the infectious uveitis we find those caused by parasitic infestations which are endemic in our country. The diagnosis is made through a good anamnesis, complete physical examination and ophthalmological assessment based on visual acuity, biomicroscopy with slit lamp and eye fundoscopy to determine the location of the parasite. There is no specific treatment for this pathology, the only effective one, although difficult and not always possible, is surgical removal of the parasite. Next, we present a clinical case of a female patient who consulted for edema of left hemiface accompanied by left periorbital edema and low vision of the left eye, to which a complete ophthalmological examination was performed and the presence of a tubular parasite was found adhered to the anterior capsule of the lens of the left eye. Medical treatment is established, and surgical removal of the parasite is performed.

KEYWORDS: uveitis, vision low, parasites.

Auveíte é a patologia do trato uveal que descreve a inflamação da úvea em seus três segmentos: anterior, médio, posterior ou panuveíte. Dentro da uveíte infecciosa, encontramos aqueles causados por infestações parasitárias endêmicas em nosso país. 0 diagnóstico faz através de uma boa anamnese, exame físico e avaliação oftalmológica com base na acuidade visual, cortou biomicroscopia de lâmpada e fundo de olho para determinar a localização do parasita. Não há tratamento específico para essa patologia, a única remoção cirúrgica eficaz, embora difícil e nem sempre possível, do parasita. Aqui apresentamos um caso clínico de uma paciente do sexo feminino que consulta edema de hemifacial esquerda acompanhada por edema periorbital esquerdo e inferior do olho esquerdo, o qual foi submetido a exame ocular completo e a presença de um parasita tubular adere ao olho cápsula da lente anterior esquerda foi encontrado. 0 tratamento médico é estabelecido e a remoção cirúrgica do parasita é realizada.

PALAVRAS-CHAVE: uveíte, baixa visão, parasitos. 
INTRODUCCIÓN

La uveítis es la patología del tracto uveal que describe la inflamación de la úvea en sus tres segmentos: anterior, medio, posterior o en su totalidad (panuveitis). 'Las causas de uveítis pueden ser: oculares propiamente dichas o asociadas a etiologías sistémicas que pueden incluir tanto enfermedades autoinmunes como infecciones. ${ }^{2}$ Dentro de las uveítis endógenas infecciosas se encuentran las causadas por infestaciones parasitarias las cuales son endémicas en nuestro país. ${ }^{1}$ Se cuenta con poca información sobre las principales causas de uveítis infeccionas a nivel nacional; el Ecuador no comparte con otros países de la región los mismos microorganismos ni su resistencia a medicamentos, por lo que los resultados de otras regiones no necesariamente son aplicables a nuestra realidad. Se describeel caso de una paciente con uveítis de origen parasitario que presentó buena evolución luego de tratamiento antihelmíntico oral y extracción quirúrgica del parásito.

\section{CASOCLÍNICO}

Se presenta un caso clínico de una paciente de sexo femenino de 36 años de edad de ocupación docente, raza mestiza, quien reside en zona urbana de la ciudad de Guayaquil. Niega antecedentes patológicos personales o alérgicos y refiere hábito alimenticio de consumir frecuentemente pescado crudo y cirugía de pterigion nasal ojo izquierdo, hace 5 años.

Acudea la emergencia por presentar cuadro clínico de 4 días de evolución caracterizado por edema de hemicara izquierda y edema periorbitario izquierdo acompañado de baja visión y enrojecimiento del ojo izquierdo. La paciente asistió por reiteradas ocasiones al servicio de emergencia general, tres meses atrás, por presentar sintomatología similar a nivel de torax posterior con lesiones dérmicas nodulares pruriginosas que luego aparecieron en región facial contralateral sin compromiso ocular; fue tratada como un cuadro de atopía; sin embargo los síntomas fueron recurrentes a pesar del tratamiento ambulatorio.

La paciente fue hospitalizada en el área de emergencia del hospital Teodoro Maldonado Carbo en septiembre del año 2015, por presentar edema de hemicara izquierda y edema periorbitario izquierdo. Le realizaron interconsulta al servicio de oftalmología en su $4^{\circ}$ dia de hospitalización, por presentar dolor ocular acompañado de baja visión de su ojo izquierdo.
Al examen físico general signos vitales dentro de límites normales, presión arterial 120/80 mmHg., temperatura corporal $37^{\circ} \mathrm{C}$., Glasgow 15/15; a la inspección general llama la atención la presencia de edema periorbitario más inyección periquerática del ojo izquierdo.

Al examen oftalmológico la agudeza visual de su ojo derecho 20/20 con $j^{1}$ y ojo izquierdo 20/300 con j no valorable, presión intraocular ojo derecho $16 \mathrm{mmHg}$, ojo izquierdo $37 \mathrm{mmHg}$. A la biomicroscopía en la lámpara de hendidura el ojo derecho se encontraba sin alteraciones; en ojo izquierdo se observa edema bipalpebral e hiperemia conjuntival, en córnea presencia de edema microquístico; cámara anterior a nivel de hora 10, siguiendo las manecillas del reloj, presencia de parásito con forma tubular que llega hasta cápsula anterior del cristalino, produciendo una reacción inflamatoria en forma de anillo como se muestra en la figura 1.

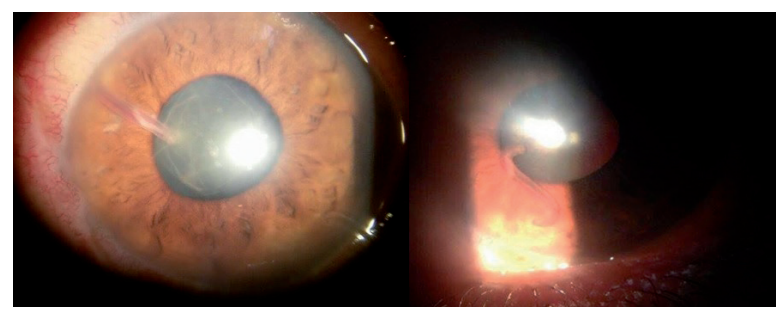

Figura 1. A.- Biomicroscopía del ojo izquierdo con lámpara de hendidura donde se observa reacción inflamatoria de la cápsula anterior del cristalino causada por la adherencia del parásito. B.- Biomicroscopía del ojo izquierdo con lámpara de hendidura donde se observa desplazamiento parcial del parásito con disminución de la reacción inflamatoria posterior al 3er día del tratamiento.

Se realizan exámenes de laboratorio generales como biometría hemática completa y coproparasitario, evidenciando leucocitos 10.280 con un porcentaje de eosinofilos $3.7 \%$. Se solicita interconsulta al servicio de infectología quienes indican realizar eco ocular, resonancia magnética del ojo afectado e iniciar tratamiento con albendazol $15 \mathrm{mg} / \mathrm{kg} /$ día, ivermectina 200 $\mathrm{mcg} / \mathrm{kg} / \mathrm{día}$ por 3 días, praziquantel $50 \mathrm{mg} / \mathrm{kg} /$ día y dexametasona $0.1 \mathrm{mg} / \mathrm{kg} /$ día (1 día antes de iniciar los antiparasitarios).

En los exámenes imagenológicos complementarios como la ecografía ocular y resonancia magnética, no se evidenciaron lesiones ni cuerpos extraños. Por lo que se decide iniciar a su vez tratamiento local con antihipertensivos oculares (timolol + brimodinina) 1 gota cada 12 horas en ojo izquierdo y esteroideos tópicos (prednisolona) 1 gota cada 4 horas en ojo izquierdo. 
Seis días después de iniciado el tratamiento descrito, se realiza gonioscopía y se observa parásito en seno camerular. Se programa paracentesis terapéutica en quirófano y se extrae parásito del seno camerular del ojo izquierdo utilizando una cánula de sincoe. (Figura 2).

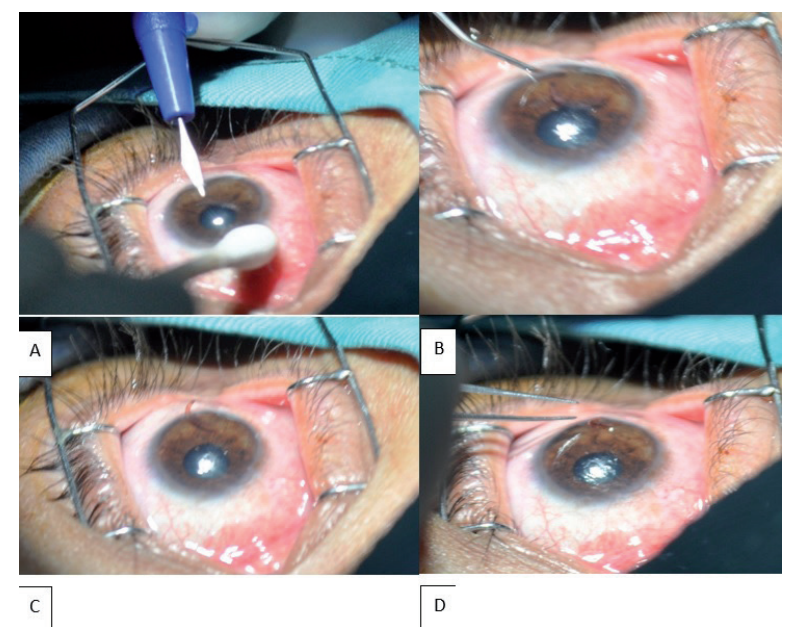

Figura 2. A.- Procedimiento quirúrgico en el cual se realiza paracentesis en limbo esclerocorneal con cuchilleta de $15^{\circ}$ en zona temporal. B.- Colocación de viscoelástico (hidroxipropilmetilcelulosa) en cámara anterior, desplazando el parásito del ángulo camerular. C.-Extracción de parásito a través de la paracentesis temporal. D.-Exposición completa del parásito sobre cornea de ojo izquierdo de la paciente.

Se envía cuerpo extraño (parásito) extraído de cámara anterior del ojo izquierdo al laboratorio clínico, patología clínica reporta que se trata de un parásito helminto.

Cinco días después de dicho procedimiento, se revalora a la paciente evidenciando una mejoría de la agudeza visual de su ojo izquierdo 20/20 j1 sin corrección y presión intraocular de ojo izquierdo de $17 \mathrm{mmHg}$. En la lámpara de hendidura se observa disminución considerable de la inflamación de la cámara anterior; sin embargo, se evidencia discretos y escasos pigmentos en la cápsula anterior del cristalino, por lo que se continuó con antiinflamatorios esteroideos tópicos (prednisolona) por 5 días más y luego se da el alta hospitalaria a la paciente. Se realiza seguimiento del caso clínico 8 semanas después del alta hospitalaria encontrando a la paciente asintomática sin recurrencia ni secuelas visuales.

\section{DISCUSIÓN}

El caso expuesto es una patología ocular poco común en nuestro medio, ya que a pesar de ser un país considerado como zona endémica de dicha parasitosis, pocas debutan con manifestaciones oculares. ${ }^{1}$

A pesar de que la muestra extraída del paciente se envió a laboratorio de patología, el resultado del estudio no proporcionó datos específicos de la etiología del parásito, sin embargo se pudo analizar a través de la lámpara de hendidura de forma macroscópica que se trata de un parasito cilíndrico de coloración parduzca de aproximadamente $1 \mathrm{~cm}$, con una masa nodular cefálica cubierto de espinas cuticulares adheridas a la cápsula anterior del cristalino; por dichas características morfológicas mencionadas se concluye que se estaría frente a un caso de Gnastostomiasis ocular., ${ }^{1,2}$

La gnatostomiasis o gnatostomosis es una enfermedad producida por nemátodos del género Gnathostoma, la cual normalmente se adquiere por comer carne cruda o poco cocinada de peces de agua dulce o anfibios infectados con larvas L3 (forma infectiva para hospedadores intermediarios, paraténicos y humanos). ${ }^{3}$

Accidentalmente afecta al hombre, en el cual los nemátodos nunca alcanzan su estado adulto (salvo alguna rara excepción), sino que son las larvas L3 las que producen la enfermedad propiamente dicha (gnatostomiasis cutánea, ocular, visceral, pulmonar, genitourinaria, neurológica). ${ }^{4,5}$

Se usó como terapéutica en este caso clínico, protocolos médicos y farmacológicos de la OMS ante este tipo de infestaciones parasitarias; además, siguiendo las indicaciones del servicio de infectología, se utilizaron 3 medicamentos simultáneamente para eliminar todas las formas evolutivas del parásito. ${ }^{6,7}$

Como antihelmíntico de primera elección se administró albendazol $15 \mathrm{mg} / \mathrm{kg} /$ día por 3 días ya que es un fármaco accesible por su eficacia y bajo costo. ${ }^{8} \mathrm{El}$ albendazol actúa dañando de forma selectiva los microtúbulos citoplasmáticos de las células intestinales de los nemátodos disminuyendo la captación de glucosa y la depleción de los depósitos de glucógeno que como consecuencia final produce autolisis del parásito. ${ }^{9}$

También se utilizó la ivermectina y praziquantel como tratamiento de elección para nematodos 
intestinales debido a su eficacia basada en producir parálisis espástica aumentando la permeabilidad de la membrana del parásito y provocando la muerte del nemátodo; además que provocan menos efectos secundarios no deseados en el paciente. ${ }^{10}$

Es importante mencionar que no existe un único tratamiento específico para esta helmintiasis, siendo la forma más efectiva, aunque difícil y no siempre posible, la extracción del gusano quirúrgicamente. ${ }^{11}$ Resulta difícil principalmente por la naturaleza migratoria de la larva o bien por su localización en órganos o tejidos diferentes de la piel. Respecto a los fármacos, se usa albendazol, mebendazol, tiabendazol, metronidazol, praziquantel, ivermectina y fenilbutazona a diferentes dosis. ${ }^{12}$ Algunos ejemplos: Ivermectina: $0,2 \mathrm{mg} / \mathrm{kg}$ en dosis única, aunque algunos autores sugieren la administración del fármaco en 2 a 3 días consecutivos. Albendazol: $400 \mathrm{mg} / 12 \mathrm{~h}$ durante 7 a 21 días.

Por las dificultades en el manejo terapéutico y complicaciones que conlleva el no tratar este tipo de infecciones a tiempo, es importante concientizar que el consumo de alimentos crudos como mariscos o pescados, puede provocar esta patología infecciosa, la cual ocasiona complicaciones severas en la salud. ${ }^{13,14}$

Estas complicaciones no solo son a nivel oftalmológico si no también pueden ser complicaciones sistémicas especialmente en países endémicos como lo es Ecuador. ${ }^{15}$

\section{CONCLUSIÓN}

Cabe resaltar la importancia de la anamnesis y de un completo examen oftalmológico a todos los pacientes que consultan con síntomas similares a los descritos en el reporte de este caso clínico. Principalmente aquellos pacientes que cursan con lesiones dérmicas, nodulares, migratorias y pruriginosas acompañadas de diversos síntomas oftalmológicos, logrando así el diagnóstico y tratamiento oportuno dando un mejor pronóstico ocular al paciente.

\section{REFERENCIAS BIBLIOGRÁFICAS}

1. Paul A, Pammal AT. Ocular parasitosis: a rare cause of hypertensive uveitis. Indian Journal Ophthalmol.2008;8:56.

2. Costa A, Bravo P, Valdez L, Levy S, Sordo C, Magill F, et al. Paniculitis nodular migratoria eosinofílica en el Perú. (Gnothostomiasis humana). Informe de once casos, polibles causas y revisión de la literatura. Fol Dermatol.2001;12(2): 21-35.

3. Cipriani V. Paniculitis nodular migratoria. Revista Universidad Peruana Cayetano Heredia.1997;10:4.

4. Sorribas M, Campos S. Protocolo de diagnóstico diferencial en el paciente con uveítis. Revista de información e investigación oftalmológica de Laboratorios Thea .2012;5:2.

5. Garcia M et al. Estudio de las Zoonosis parasitarias de localización ocular en el Instituto de Oftalmología. Revista de Investigacion de Perú. 2002;4:13.

6. Llorenç V et al. Caracterización de las uveítis diagnosticadas en un centro de referencia del área de Barcelona.. Revista Medicina Clínica de Barcelona.2012;7:138.

7. Papo M, Bielefeld P et al. Tocilizumab in severe and refractory non-infectious uveitis. Revista Reumatología Clínica y Experimental. 2014;17:32.

8. Barry RJ, Denniston AK. Controversies in the Pharmacological Treatment of Uveitis. Curr Pharm Des. 2015;9:21.

9. Egwuagu CE, Sun L, Kim SH, Dambuza IM. Ocular Inflammatory Diseases: Molecular Pathogenesis and Immunotherapy. Curr Mol Med. 2015;10:15.

10. Li CQ, Cho AA, Edward NJ, Edward DP, Fajardo RG, Mafee MF. Magnetic resonance imaging of uveitis. Neuroradiology. 2015;6:57.

11. Massilamany C, Gangaplara A, Reddy J.Environmental microbes and uveitis: is microbial exposure always bad?. Scand J Immunol. 2015;13:81.

12. Fernandez P. Uveitis Anterior Aguda. Revista médica de costa rica Y centroamérica LXVIII. 2001;11:597.

13. Sana $S$ et al.Glaucoma and Uveitis. Survey ophthalmology. 2013;58:1.

14. Taylor S et al. A case of recurrent bilateral uveitis independently associated with dabrafenib and pembrolizumab therapy. American Journal of Ophthalmology Case Reports. 2016;6:2.

15. Mustafa M. Uveitis: Pathogenesis, presentations and treatment.IOSR Journal of Pharmacy. 2012;22:50 